\title{
A multi-state coarse grained modeling approach for an intrinsically disordered peptide
}

Farhad Ramezanghorbani, Cahit Dalgicdir, and Mehmet Sayar

Citation: The Journal of Chemical Physics 147, 094103 (2017); doi: 10.1063/1.5001087

View online: https://doi.org/10.1063/1.5001087

View Table of Contents: http://aip.scitation.org/toc/jcp/147/9

Published by the American Institute of Physics

\section{Articles you may be interested in}

Transferable coarse-grained model for perfluorosulfonic acid polymer membranes

The Journal of Chemical Physics 147, 094904 (2017); 10.1063/1.4986287

Multisequence algorithm for coarse-grained biomolecular simulations: Exploring the sequence-structure relationship of proteins

The Journal of Chemical Physics 147, 095102 (2017); 10.1063/1.4986933

Extending the range and physical accuracy of coarse-grained models: Order parameter dependent interactions

The Journal of Chemical Physics 147, 044113 (2017); 10.1063/1.4995946

Dissipative particle dynamics: Systematic parametrization using water-octanol partition coefficients

The Journal of Chemical Physics 147, 094503 (2017); 10.1063/1.4992111

A coherent discrete variable representation method on a sphere

The Journal of Chemical Physics 147, 094101 (2017); 10.1063/1.4996891

Perspective: Dissipative particle dynamics

The Journal of Chemical Physics 146, 150901 (2017); 10.1063/1.4979514

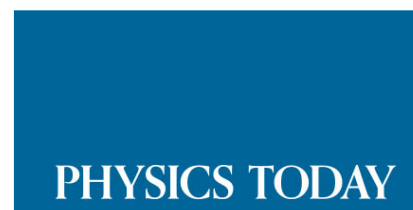

WHITEPAPERS
ADVANCED LIGHT CURE ADHESIVES

Take a closer look at what these environmentally friendly adhesive systems can do
READ NOW

PRESENTED BY

Q. MASTERBOND' 


\title{
A multi-state coarse grained modeling approach for an intrinsically disordered peptide
}

\author{
Farhad Ramezanghorbani, ${ }^{1}$ Cahit Dalgicdir, ${ }^{1}$ and Mehmet Sayar ${ }^{1,2}$ \\ ${ }^{1}$ College of Engineering, Koç University, 34450 Istanbul, Turkey \\ ${ }^{2}$ Chemical and Biological Engineering and Mechanical Engineering Departments, College of Engineering, \\ Koç University, 34450 Istanbul, Turkey
}

(Received 28 April 2017; accepted 18 August 2017; published online 6 September 2017)

\begin{abstract}
Many proteins display a marginally stable tertiary structure, which can be altered via external stimuli. Since a majority of coarse grained (CG) models are aimed at structure prediction, their success for an intrinsically disordered peptide's conformational space with marginal stability and sensitivity to external stimuli cannot be taken for granted. In this study, by using the LK $\alpha 14$ peptide as a test system, we demonstrate a bottom-up approach for constructing a multi-state CG model, which can capture the conformational behavior of this peptide in three distinct environments with a unique set of interaction parameters. $\mathrm{LK} \alpha 14$ is disordered in dilute solutions; however, it strictly adopts the $\alpha$-helix conformation upon aggregation or when in contact with a hydrophobic/hydrophilic interface. Our bottom-up approach combines a generic base model, that is unbiased for any particular secondary structure, with nonbonded interactions which represent hydrogen bonds, electrostatics, and hydrophobic forces. We demonstrate that by using carefully designed all atom potential of mean force calculations from all three states of interest, one can get a balanced representation of the nonbonded interactions. Our CG model behaves intrinsically disordered in bulk water, folds into an $\alpha$-helix in the presence of an interface or a neighboring peptide, and is stable as a tetrameric unit, successfully reproducing the all atom molecular dynamics simulations and experimental results. Published by AIP Publishing. [http://dx.doi.org/10.1063/1.5001087]
\end{abstract}

\section{INTRODUCTION}

For proteins and peptides, the conformational heterogeneity and the structural dynamics in response to external stimuli play a key role in their functionality and adsorption and aggregation behavior. With the advance of experiments capable of tracing the conformational characteristics and dynamics at a single molecule level, our understanding of these complex phenomena is developing rapidly. ${ }^{1-4}$

All atom (AA) and coarse grained (CG) computer simulations complement these single molecule experiments and further enhance our understanding of the conformational properties of proteins/peptides by providing a virtual test environment with subnanometer resolution. Despite the development of novel simulation techniques and rapid enhancements in computer architecture, state-of-the-art AA simulations still face limitations reaching the experimentally relevant length and time scales in many cases. ${ }^{5}$ Simulations with CG models are frequently used to bridge the gap in length and time scale between the AA simulations and experiments. For a recent review of the state-of-the-art CG models for proteins/peptides, see the perspective article by Noid. ${ }^{6}$ A major challenge in the development of CG models is to maintain transferability both in terms of sequence and thermodynamic properties. To this aim, several CG models have been proposed in recent years, which target different aspects of the general transferability problem, including but not limited to UNRES, ${ }^{7}$ MARTINI, ${ }^{8}$ OPEP, ${ }^{9}$ PLUM,${ }^{10}$ PRIMO,${ }^{11}$ and PRIME. ${ }^{12}$ The article by Kar and Feig ${ }^{13}$ provides a comprehensive review and comparison of these models with regard to transferability. With the ever expanding efforts towards understanding intrinsically disordered proteins/peptides, the development of transferable CG force fields that are not biased towards a particular secondary structure and able to represent rich conformational space of such peptides became even more important. ${ }^{14}$

The general approach in the design of transferable CG models can be grouped into two broad categories. The first group of CG models aims to create a sequence independent generic force field. ${ }^{8,10,12,15,16}$ The widely used Martini force field is a perfect example of this category and relies on the reproduction of solvation free energies. A possible downside of these generic potentials is a priori selection of the secondary structure as in the case of Martini. Alternatively, one can rely on knowledge-based potentials for targeting structural and/or thermodynamic properties as demonstrated by Prime $20^{12}$ and the model by Bereau and Deserno. ${ }^{10}$

The second category includes CG models which aim to represent the changes in structure and/or thermodynamic properties of the target molecules in response to changes in environment such as temperature, $\mathrm{pH}$, salt, and peptide concentration or introduction of an interface. ${ }^{17-28}$ Bottom-up CG models hold a great premise in this respect. Either by optimizing potentials via taking into account more than one state point ${ }^{20,24}$ or by introducing a built-in dependence on the respective state point changes, ${ }^{18,19}$ one can construct transferable CG models. 
Our CG model falls into this second category. Here, by using a model peptide composed of only two types of residues, we will demonstrate our approach in developing a transferable CG model which can represent both the conformational dynamics and the association behavior of the target system in three different thermodynamic states.

Peptides composed of only two types of residues, leucine and lysine, have been widely utilized in both experimental and computational studies as model systems for studying the interplay between conformation and adsorption and aggregation behavior. ${ }^{29-36}$ LK $\alpha 14$ molecule, designed by DeGrado and Lear, ${ }^{29}$ was part of a systematic study on induction of peptide conformation in bulk and at apolar/water interfaces. This molecule, designed to display the secondary amphiphilic character in the $\alpha$-helix conformation, does not have a unique conformation below the critical micelle concentration (CMC) in bulk water. ${ }^{29,35,36}$ The stabilization of the $\alpha$-helix conformation can be achieved either by increasing the concentration above the CMC (which triggers the formation of four-helix bundles) or by exposing the system to hydrophobic/hydrophilic surfaces. ${ }^{29,30,35,36}$

In this study, our goal is to develop a multi-state CG model which can represent the conformation and aggregation and adsorption behavior of $\mathrm{LK} \alpha 14$ in three different states with a unique set of interaction potentials. To this aim, we used all atom molecular dynamics (MD) simulations for $\mathrm{LK} \alpha 14$ from our earlier study ${ }^{36}$ as reference states for the parameterization of the nonbonded interaction potentials. We construct our CG model by combining a base model for bonded interactions, that is not biased towards any particular secondary structure, with nonbonded interactions that are parameterized with respect to the AA potential of mean force (PMF) curves from three distinct environments. This approach enables us to capture the delicate balance between the nonbonded interaction terms, which results in a fairly accurate representation of the degenerate conformational space in bulk water.

In the following, we first describe the model and parameterization of bonded and nonbonded interaction potentials. Next, we test the performance of the multi-state CG model in the respective environments in terms of structural characteristics, conformational preference, and finally folding and aggregation and adsorption behavior. It is important to note that our final CG model will still be limited in transferability, i.e., we are not targeting transferability over a temperature range or sequence wise transferability in the current study.

\section{METHODS}

CG molecular dynamics (MD) simulations were performed with GROMACS version 4.5. $6^{37}$ with the leap-frog stochastic dynamics integrator and a 2 fs time step. MD simulations were performed in the canonical ensemble (NVT). Temperature was set to $298 \mathrm{~K}$ using the velocity-rescaling algorithm $^{38}$ with a coupling time constant of 1 ps. The van der Waals (VDW) interactions were truncated at $1.0 \mathrm{~nm}$. Long range electrostatic interactions were calculated with the particle mesh Ewald (PME) ${ }^{39}$ method with a Coulomb cutoff of $2.0 \mathrm{~nm}$ and Fourier spacing of $0.24 \mathrm{~nm}$.
All atom (AA) MD simulations for $\mathrm{LK} \alpha 14$, as discussed in the work of Dalgicdir and Sayar, ${ }^{36}$ were used as reference systems for the parameterization of the CG model. These simulations were performed with Gromacs version $4.5 .6^{37}$ by using the GROMOS 54a7 force-field ${ }^{40}$ and explicit SPC/E water. ${ }^{41}$ Further details of the simulations can be found in the study by Dalgicdir and Sayar. ${ }^{36}$ The VOTCA toolkit ${ }^{42}$ has been used to map the all atom reference trajectories onto the CG model for the $\operatorname{LK} \alpha 14$ peptide.

The umbrella sampling ${ }^{43}$ method was used for potential of mean force (PMF) ${ }^{44}$ calculations in three different cases: splitting up a dimer of $\mathrm{LK} \alpha 14 \mathrm{~s}$ in bulk water, stretching of an $\operatorname{LK} \alpha 14 \alpha$-helix, and finally for adsorption of an LK $\alpha 14$ molecule to the air/water interface. Each umbrella window was simulated for at least $200 \mathrm{~ns}$. The final potential of mean force (PMF) curves were obtained by using the weighted histogram analysis method (WHAM) ${ }^{45}$ as implemented in the g_wham toolkit $^{46}$ of GROMACS. The convergence for the PMF curves was validated by block analysis.

For the dimer umbrella simulation, the center of mass of the backbone beads of the peptides was used as the pull and reference groups. A pulling rate of $0.005 \mathrm{~nm} / \mathrm{ps}$ was used with a force constant of $1000 \mathrm{~kJ} /\left(\mathrm{mol} \times \mathrm{nm}^{2}\right)$. For stretching the $\alpha$-helix, the CA beads at the two ends were used as pull and reference groups. A pulling rate of $0.01 \mathrm{~nm} / \mathrm{ps}$ and a force constant of $1000 \mathrm{~kJ} /\left(\mathrm{mol} \times \mathrm{nm}^{2}\right)$ were used. Finally, for pulling the peptide from the interface, the center of mass of the backbone beads and a dummy particle attached to the wall was used as the pull and reference groups. Pulling was performed along the $\mathrm{z}$ direction perpendicular to the interface with a pulling rate of $0.005 \mathrm{~nm} / \mathrm{ps}$ and a force constant of $1000 \mathrm{~kJ} /\left(\mathrm{mol} \times \mathrm{nm}^{2}\right)$.

Hamiltonian replica exchange ${ }^{47}$ (HREX) as implemented in GROMACS 4.6.148 patched with the PLUMED plugin ${ }^{49}$ version 2.2.0 has been used to enhance the sampling of conformational space of the CG model. 16 replicas were set up for the HREX simulations. The replicas differed for the strength of the hydrogen bond interaction term, where the depth of the interaction potential was set to cover the range of $14-17.75 \mathrm{~kJ} / \mathrm{mol}$ with $0.25 \mathrm{~kJ} / \mathrm{mol}$ intervals. The HREX simulation was conducted for $2 \mu \mathrm{s}$, in implicit solvent with NVT ensemble. A conformation exchange between neighboring replicas was attempted every 1000 steps and the average exchange probability was calculated as 0.84 .

For comparing the resulting HREX trajectories with the AA temperature $\mathrm{REX}^{36}$ simulations, a cluster analysis was performed by using the g_cluster tool of Gromacs. We selected random conformations from AA temperature $\mathrm{REX}^{36}$ from $\mathrm{T}=298 \mathrm{~K}$ and CG HREX simulations for $\varepsilon_{h b}=17(12500$ frames from each). After mapping the AA trajectory to the CG representation, we merged the two sets of frames. The trajectory with 25000 frames was clustered by using the g_cluster tool of Gromacs. The Jarvis-Patrick clustering algorithm with a cutoff distance of $0.14 \mathrm{~nm}$ was used for the clustering. The resulting clusters were analyzed to obtain the fraction of AA and CG frames in each. 16 clusters with more than 100 frames were selected and further grouped into one of three dominant conformations observed: $\alpha$-helix, half- $\alpha$-helix, and $\beta$-hairpin. The remaining frames that were not included in any one of these clusters were considered as random. 
The molecular renderings in all figures were produced by using VMD..$^{50}$

\section{CG MODEL}

\section{A. Mapping}

The $\mathrm{LK} \alpha 14$ peptide is composed of leucine $(\mathrm{L})$ and lysine (K) residues with a sequence of Ace-(LKKLLKL) 2 -Nme, where Ace and Nme are capping groups to eliminate the terminal charges. For the CG representation of the LK $\alpha 14$ molecule, the mapping scheme shown in Table I is used. Both the AA and CG representations of the molecule are shown in an ideal $\alpha$-helix conformation in Fig. 1. In our model, the backbone of the peptide is represented by two beads, $\mathrm{CN}$ and CA, which allows us to represent two backbone dihedral angles to capture the conformational flexibility of the backbone. Similar two bead backbone models were successfully used for studying $\beta$-sheet type aggregation 27,51 and helix-coil transitions ${ }^{26}$ by peptide CG models. In order to represent the backbone hydrogen bonding capacity of the molecule, a virtual bead (represented by $\mathrm{H}$ ) is used.

Representation of the leucine side chain with a single bead $\mathrm{L}$ yields a probability distribution with a single peak for the CA-L bond length (see Fig. S1 of the supplementary material). The lysine side chain is much longer and is composed of two distinct groups in terms of charge: one neutral and the other carrying a +1 charge. Therefore, we represented the lysine side chain by a neutral bead (KC) and a charged bead $(\mathrm{KN})$.

Chloride counterions are used to maintain charge neutrality and are mapped as CL beads. The mass of each bead type and the required number of beads from each type to represent a single CG molecule are also shown in the table. All beads are mapped onto the center of mass of the group of atoms they represent. The position of the virtual site hydrogen bead $(\mathrm{H})$ is calculated based on the $\mathrm{CN}$ bead and the two neighboring CA beads according to the following equation: ${ }^{52}$

$$
r_{s}=r_{i}+a r_{i j}+b r_{i k}+c\left(r_{i j} \times r_{i k}\right),
$$

where $r_{s}, r_{i}, r_{j}$, and $r_{k}$ are the positions of the virtual site $\mathrm{H}_{i}, \mathrm{CN}_{i}, \mathrm{CA}_{i-1}$, and $\mathrm{CA}_{i}$ beads, respectively. The constants $a, b$, and $c$ are fitted to an ideal $\alpha$-helix structure, such that the virtual hydrogen bead is located at the center of the line connecting beads $\mathrm{CN}_{i}$ and $\mathrm{CN}_{i+3}$ as $a=1.8167, b=1.6320$, and $c=1.6503$.

TABLE I. CG bead types, list of atoms used for the mapping, mass of the bead type, and the required number of beads of each type for representation of a single $\mathrm{LK} \alpha 14$ peptide.

\begin{tabular}{lccc}
\hline \hline Beads & Atoms & Mass & No. of beads \\
\hline $\mathrm{CN}$ & $\mathrm{C}, \mathrm{O}, \mathrm{N}, \mathrm{H}$ & 43.03 & 16 \\
$\mathrm{CA}$ & $\mathrm{C}_{\alpha}$ & 13.02 & 15 \\
$\mathrm{H}$ & Virtual site & 0 & 15 \\
$\mathrm{~L}$ & $\mathrm{C}_{\beta}, \mathrm{C}_{\gamma}, \mathrm{C}_{\sigma 1}, \mathrm{C}_{\sigma 2}$ & 57.116 & 6 \\
$\mathrm{KC}$ & $\mathrm{C}_{\beta}, \mathrm{C}_{\gamma}, \mathrm{C}_{\sigma 1}$ & 42.081 & 8 \\
$\mathrm{KN}$ & $\mathrm{C}_{\eta}, \mathrm{NH}_{3}$ & 42.08 & 8 \\
$\mathrm{CL}$ & $\mathrm{Cl}$ & 35.45 & 8 \\
\hline \hline
\end{tabular}
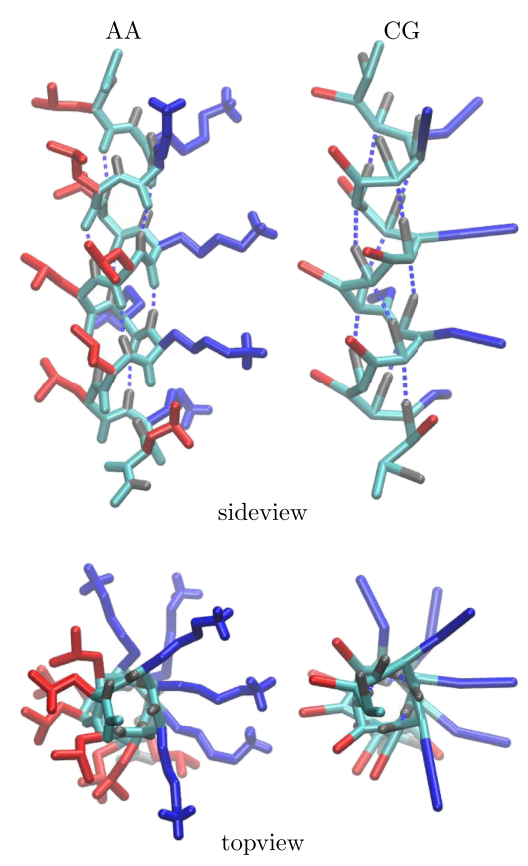

FIG. 1. The AA (left) and CG (right) representation of the LK $\alpha 14$ peptide in an $\alpha$-helix conformation. The side view and top view of the peptide are shown. The color scheme for the CG beads: $\mathrm{L}-$ red, $\mathrm{KC}$ and $\mathrm{KN}-$ blue, backbone beads $(\mathrm{CA}$ and $\mathrm{CN})$ - cyan, and $\mathrm{H}$ - gray. In the AA representation, group of heavy atoms mapped onto a CG bead (as in Table I) are colored identical to the CG case. Hydrogen bonds are shown with dotted blue lines.

\section{B. Hamiltonian}

The Hamiltonian of the CG model is formulated as two separate terms for the bonded and the nonbonded interactions,

$$
U^{c g}=U_{\text {bonded }}^{c g}+U_{\text {nonboned }}^{c g},
$$

where $U^{c g}$ is the total CG interaction potential. The separation of the bonded and nonbonded interactions enables us to parametrize them independently, which will be described in Subsections III C and III D. A summary of all bonded and nonbonded interaction potentials for the final CG model is provided in Sec. II of the supplementary material.

\section{Bonded interactions}

We used the Boltzmann inversion (BI) method ${ }^{53,54}$ to obtain the interaction potentials for the bond length $\left(U_{r}^{c g}\right)$, bond angle $\left(U_{\theta}^{c g}\right)$, and dihedral angle $\left(U_{\phi}^{c g}\right)$ stiffness terms, which add up to the $U_{\text {bonded }}^{c g}$ term in Eq. (2),

$$
U_{\text {bonded }}^{c g}=U_{r}^{c g}+U_{\theta}^{c g}+U_{\phi}^{c g} .
$$

The reference probability distributions for these bonded interactions are obtained by mapping the AA simulation of the molecule onto the CG model and by analyzing the distributions of the relevant degrees of freedom. We have analyzed the probability distributions for a single molecule in four different environments: (1) in vacuum, (2) in bulk water, (3) within an aggregate of four $\operatorname{LK} \alpha 14 \mathrm{~s}$ (tetramer), and (4) at the air/water interface. The aim of the simulation in vacuum is to obtain the probability distribution of the bonded interactions without the bias introduced by the long range nonbonded intraor inter-molecular interactions, as well as the solvent or an 
air/water interface. Therefore, for the vacuum simulation, the nonbonded interactions are turned off, except for atom pairs that are mapped onto CG beads connected via any one of the $\mathrm{CG}$ bonded interaction terms. In addition, the dielectric constant is set to $\varepsilon=78$ to prevent the bias from strong Coulomb interactions in vacuum.

In the AA vacuum simulation, in the absence of explicit water and nonbonded interactions, the molecule adopts random conformations. The conformational behavior of the molecule in AA simulations in bulk water, within a tetramer and at the air/water interface, was investigated in our earlier studies. ${ }^{35,36}$ In bulk water, the conformation of the LK $\alpha 14$ peptide alternates between an $\alpha$-helix, a half $\alpha$-helix, and a $\beta$ hairpin. On the other hand, in the presence of the neighboring molecules within the tetramer and at the air/water interface, the amphiphilic nature of the molecule favors a full $\alpha$-helix and all other conformations are eliminated.

The resulting probability distributions from these reference states for bond stretching, angle bending, dihedral, and side chain improper dihedrals are shown in Figs. S1$\mathrm{S} 4$ of the supplementary material. The backbone dihedral angle probability distributions, which will be used for $\alpha$ helix characterization, are also shown in Figs. 2(a) and 2(b). Among the three bonded interaction types, dihedral distributions display the strongest dependence on the simulation environment. In comparison, bond length and bond angle distributions are only modestly altered in all four cases considered. Since the tetramer and interface simulations yield similar results, only the tetramer simulations are discussed in the following.

As the bond and angle distributions display a weak dependence on the environment, their parameterization can be based on any one of the AA reference states, without altering the representability of the CG model. For the bond length stretching interaction, a harmonic fit is performed on the tetramer probability distributions. Some of the resulting spring constants are too stiff and enforce a time step of 2 fs for an adequate sampling of the fluctuations. ${ }^{52,55}$ In order to enable a higher time step, we softened these spring constants so that they allow a 4 fs time step. The resulting equilibrium bond length and stiffness values are provided in Sec. II of the supplementary material.

For the bond angle terms, tabulated potentials are used (shown in Sec. II, Fig. S5, of the supplementary material), which are derived by the Boltzmann inversion of the AA probability distributions obtained from the tetramer reference system (provided as Sec. I, Fig. S2, of the supplementary material). In total, seven tabulated angle potentials are used: two for the backbone $(\mathrm{CA}-\mathrm{CN}-\mathrm{CA}$ and $\mathrm{CN}-\mathrm{CA}-\mathrm{CN})$ and five for the side chain angles $(\mathrm{CN}-\mathrm{CA}-\mathrm{L}, \mathrm{L}-\mathrm{CA}-\mathrm{CN}$, $\mathrm{CN}-\mathrm{CA}-\mathrm{KC}, \mathrm{KC}-\mathrm{CA}-\mathrm{CN}$, and $\mathrm{CA}-\mathrm{KC}-\mathrm{KN})$.

Unlike the bond stretching and angle bending distributions, the dihedral angle probability distributions strongly depend on the environment. In Fig. 2, probability distributions are shown for vacuum, bulk water, and tetramer AA reference states. The two backbone dihedral angles among the $\mathrm{CA}-\mathrm{CN}-\mathrm{CA}-\mathrm{CN}$ and $\mathrm{CN}-\mathrm{CA}-\mathrm{CN}-\mathrm{CA}$ beads are shown in (a) and (b), respectively. In analogy with the peptide dihedral angles, we will refer to the $\mathrm{CA}-\mathrm{CN}-\mathrm{CA}-\mathrm{CN}$

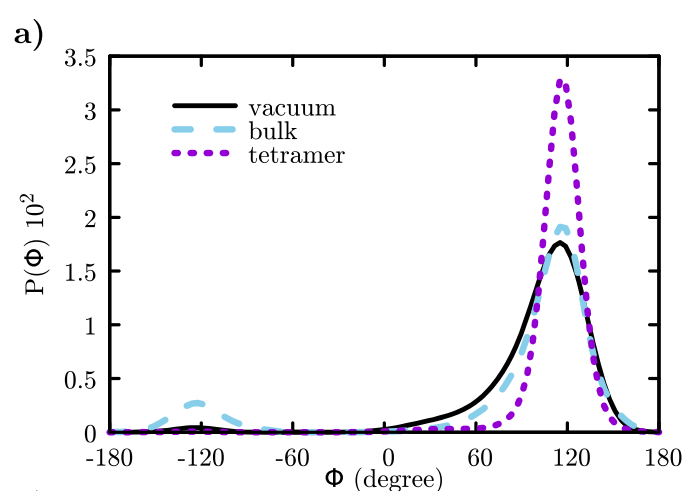

b)
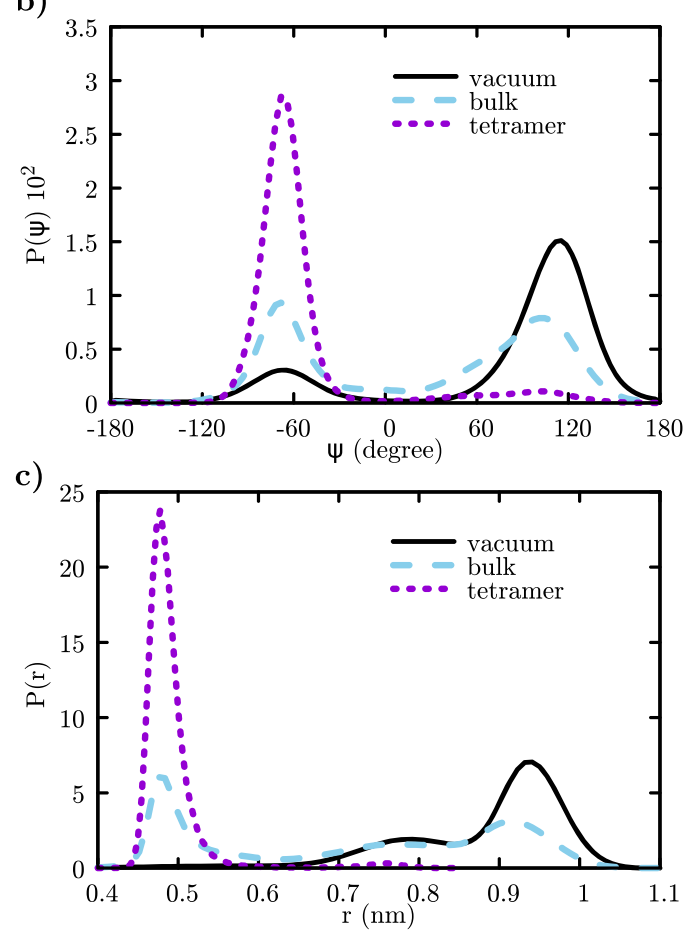

FIG. 2. AA reference probability distributions for the backbone dihedral angle $\mathrm{CA}-\mathrm{CN}-\mathrm{CA}-\mathrm{CN}$ (a) and $\mathrm{CN}-\mathrm{CA}-\mathrm{CN}-\mathrm{CA}$ (b). The probability distribution for the $\mathrm{CN}_{i+3}-\mathrm{CN}_{i}$ distances, which represent the hydrogen bonds that stabilize the $\alpha$-helix conformation (c).

dihedral as $\phi$ and the $\mathrm{CN}-\mathrm{CA}-\mathrm{CN}-\mathrm{CA}$ dihedral as $\psi$. Note that due to the CG mapping, the sign for the $\phi$ angle is reversed in our CG model with respect to the Ramachandran map convention. For the side chains, we only used the improper dihedral angle potentials and omitted the dihedral angles which include the side chain beads, in order to avoid creating an overdetermined system.

Another well defined characteristic signature of the $\alpha$ helix conformation is the hydrogen bond pattern between the $i+4$ th $\rightarrow i$ th residues. In our CG model, this bonding pattern corresponds to an interaction between $i+3 \mathrm{rd} \rightarrow i$ th residues since the $\mathrm{CN}$ bead includes both the $\mathrm{C}=\mathrm{O}$ and $\mathrm{N}-\mathrm{H}$ groups. The probability distribution for the length of this bond is also shown in Fig. 2(c).

We will use the $\mathrm{CN}-\mathrm{CA}-\mathrm{CN}-\mathrm{CA}$ dihedral angle $(\psi)$ and the $\mathrm{CN}_{i+3} \rightarrow \mathrm{CN}_{i}$ distance to analyze the $\alpha$-helix content in the molecule. As seen in Figs. 2(b) and 2(c), these two measures correctly distinguish the random conformation in vacuum (solid black line), partially $\alpha$-helical state in bulk water 
(dashed blue line), and full $\alpha$-helix in tetramer (dotted purple line) states.

Since the dihedral angle distributions strongly depend on the environment, the conformational behavior of the molecule cannot be represented in the CG model simply by adding a dihedral angle potential. The transitions observed in the AA reference states depend on the nonbonded interactions which stem from the surrounding medium. Hence, in our CG model, we will also use the combination of dihedral potentials and nonbonded interactions to capture the dynamic conformational behavior of the $\mathrm{LK} \alpha 14$ peptide. To this aim, the backbone dihedral and the side chain improper potentials are obtained from the Boltzmann inversion of the AA vacuum probability distributions, where the resulting potentials are shown in Figs. S6a and S6b of the supplementary material, respectively.

\section{Non-bonded interactions}

The secondary structure of the CG LK $\alpha 14$ peptide model, as well as its aggregation in bulk water and adsorption to a hydrophobic/hydrophilic interface, depends on the interplay of three non-bonded interactions: Coulomb interactions due to charged lysine side chains, hydrophobic interactions due to leucine side chains, and backbone hydrogen bonds. We relied on three reference states for the parameterization of these non-bonded interactions: a single molecule in bulk water, aggregation of two $\mathrm{LK} \alpha 14$ peptides in bulk water, and finally adsorption of the $\operatorname{LK} \alpha 14$ peptide to the vacuum/water interface. In Subsections III D 1-III D 4, parameterization of these three nonbonded interactions will be discussed. For all the remaining nonbonded pair interactions between CG beads, to represent excluded volume interactions, a generic repulsive Lennard-Jones (LJ) interaction is used,

$$
V(r)=4 \varepsilon_{r}\left[\left(\frac{\sigma}{r}\right)^{12}-\left(\frac{\sigma}{r}\right)^{6}+\frac{1}{4}\right] \quad 0 \leq r \leq r_{\text {cut }},
$$

where $\varepsilon_{r}=1.0 \mathrm{~kJ} / \mathrm{mol}$ and $r_{\text {cut }}=2^{\frac{1}{6}} \sigma=0.2 \mathrm{~nm}$.

\section{Electrostatics}

The LK $\alpha 14$ peptide has six charged lysine side chains (represented by $\mathrm{KN}$ beads) and six chloride counterions (represented by CL beads) to maintain charge neutrality. Remaining CG beads do not carry any charges. Water molecules are represented implicitly via a continuum dielectric constant.

In Fig. 3, the radial distribution function (RDF) for the $\mathrm{KN}$ and CL beads are compared for AA and CG simulations of a single $\mathrm{LK} \alpha 14$ peptide in bulk water. When the generic $\mathrm{LJ}$ repulsion is used for the positive and negative beads, the ions can penetrate to distances as close as $0.2 \mathrm{~nm}$ (dashed blue line). In order to mimic the AA case, we have modified the LJ repulsion between $\mathrm{KN}$ and CL beads by setting $\varepsilon_{r}=10 \mathrm{~kJ} / \mathrm{mol}$ and $r_{c u t}=0.358 \mathrm{~nm}$. With the modified LJ repulsive interaction, the CG RDF matches the first peak of the AA case (dotted purple line). Further characteristic peaks observed in the AA RDF, associated with the hydration layers around the ions, are not

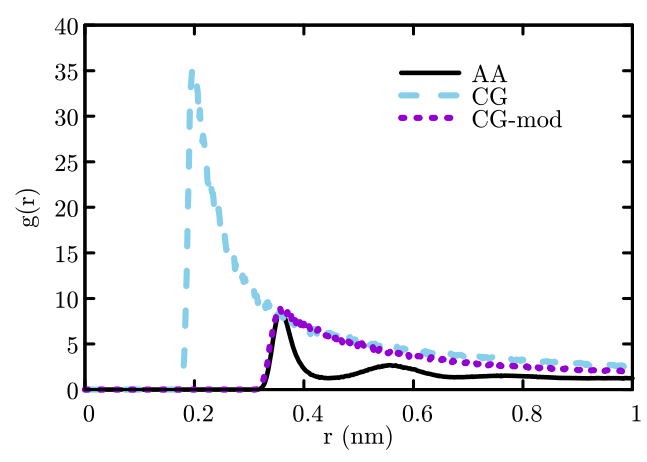

FIG. 3. RDF for KN and CL beads for a single LK $\alpha 14$ peptide in bulk water: AA (solid black line), CG with generic LJ repulsion (dashed blue line), and CG with modified LJ interaction parameters to match the AA case (dotted purple line).

present in the CG case due to the implicit solvent nature of the model.

\section{Hydrophobic forces}

The LK $\alpha 14$ peptide forms a tetramer in bulk water, ${ }^{29,36}$ where hydrophobicity is the main driving force for aggregation. Due to the molecule's secondary amphiphilic nature, in the $\alpha$-helix conformation, all leucine side chains are located on one side of the helix. In order to shield this large hydrophobic patch from water, LK $\alpha 14$ molecules readily aggregate. Since, we have an implicit water CG model, the only way to represent the hydrophobic forces is via an attractive interaction among leucine beads.

In order to mimic the short range nature of the hydrophobic interactions, we used an LJ-cosine interaction potential, ${ }^{56}$

$$
U= \begin{cases}4 \varepsilon_{L}\left[\left(\frac{\sigma}{r}\right)^{12}-\left(\frac{\sigma}{r}\right)^{6}\right], & r \leq r_{m}, \\ -\varepsilon_{L}\left[\cos \left(\frac{\pi\left(r-r_{m}\right)}{2\left(r_{\text {cut }}-r_{m}\right)}\right)\right]^{2}, & r_{m}<r<r_{\text {cut }}, \\ 0, & r_{\text {cut }} \leq r,\end{cases}
$$

where $r_{m}=2^{\frac{1}{6}} \sigma$. This potential enables a short range interaction and ensures that the force goes to zero at the cutoff distance. The RDF of leucine side chains in the AA tetramer simulation (shown in Fig. 4) is used to determine

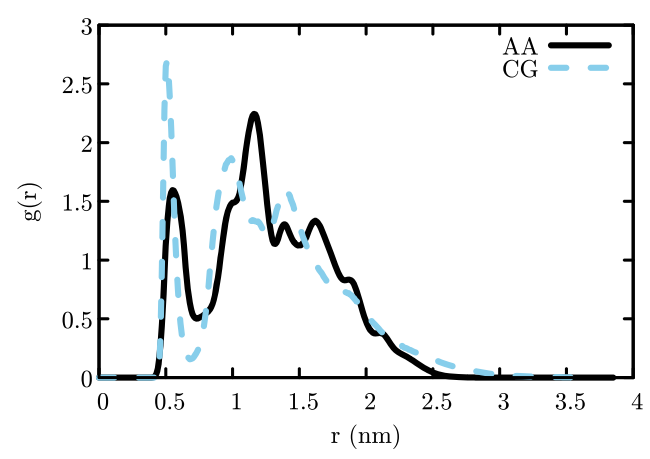

FIG. 4. RDF for the leucine side chain beads, L, in AA (solid black line) and $\mathrm{CG}$ (dashed blue line) simulations with $\varepsilon_{L}=8.5 \mathrm{~kJ} / \mathrm{mol}$ for the $\mathrm{L}-\mathrm{L}$ interaction strength. The RDF curves are not normalized for increasing distance, but rather scaled to reflect the average number of beads at a given distance $r$. 
the potential minimum $\left(r_{\text {min }}\right)$ and cutoff $\left(r_{c u t}\right)$ distances as $0.507 \mathrm{~nm}$ and $0.7 \mathrm{~nm}$, respectively. The position of the minimum corresponds to the maximum of the first peak and the cutoff distance is chosen as the minimum between the first two peaks in the RDF. Note that the RDF in Fig. 4 is not normalized and scaled such that the $y$-axis corresponds to the average number of neighbors for the distance $r$.

The Boltzmann inversion of the normalized RDF curve is shown in Fig. S7 of the supplementary material as a solid black line. This cannot be used directly for two reasons: First, the AA simulation is not a bulk simulation for leucine side chains and the resulting RDF only reflects the neighbors within the aggregate. Second, due to the correlations, the depth of the BI potential is largely overestimated. One possible solution to the latter problem is to apply iterative BI. ${ }^{57}$ However, since we are interested in not only structural but also dynamic and aggregation properties of the CG model, we will use an alternative approach.

In order to capture the assembly characteristics of the LK $\alpha 14$ peptide, we used the AA potential of mean force (PMF) curve for the parameterization of the leucine $\mathrm{LJ}$-cos potential. In our earlier study, ${ }^{36}$ the PMF curve for splitting a pair of LK $\alpha 14 \alpha$-helices was calculated via umbrella sampling technique for the AA case. The corresponding AA PMF curve is shown in Fig. 5 as a solid black line. The well defined minimum corresponds to the dimer state where both molecules are in the $\alpha$-helix conformation. As the peptides are pulled apart, the molecules partially unfold to maintain hydrophobic side chain contacts. The repulsive regime beyond $2.6 \mathrm{~nm}$ stems from the long range Coulomb repulsion among the two LK $\alpha 14$ peptides. In the repulsive regime, the leucine side chains of the peptides are completely separated from each other.

In order to tune the $\varepsilon_{L}$ parameter in Eq. (5), a similar umbrella sampling simulation to the AA is performed to obtain the CG PMF curve for the dimer. Since the potential term for representing the hydrogen bonding is not parameterized at this point, a harmonic bond is used to restrain all $\mathrm{H}_{i+3}-\mathrm{CN}_{i}$ pair distances. These harmonic bonds ensure that the $\alpha$-helix conformation is maintained during the umbrella sampling simulation. This changes the simulation setup compared to the AA

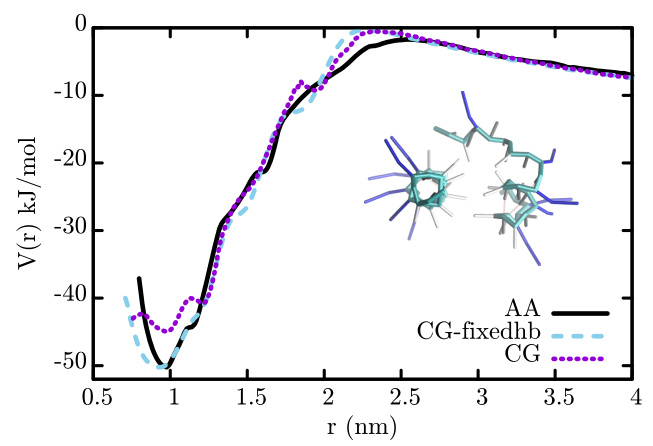

FIG. 5. PMFs for splitting a dimer of LK $\alpha 14$ peptides: AA reference (solid black line), $\mathrm{CG}$ simulation with $\varepsilon_{L}=8.5 \mathrm{~kJ} / \mathrm{mol}$ with restraints to maintain the $\alpha$-helix hydrogen bonds (dashed blue line), and CG simulation with the final model without the restraints (dotted purple line). Unfolding of the molecule, as shown in the inset, leads to the mismatch between AA and CG PMF curves at close distances. case (where the molecules are free to fold/unfold) and should be verified after the parameterization of the hydrogen bonding interaction. The electrostatic interactions are calculated via the PME method as discussed in Sec. III D 1.

Since the LJ-cos interaction is isotropic, the first and second nearest neighbor L beads within the molecule also strongly interact and distort the conformation unphysically. To overcome this, the nonbonded interactions between the $\mathrm{L}$ beads and their first two nearest L neighbors within the molecule were excluded.

After a few iterations with different depths for the leucine LJ-cos interaction, we reproduced the AA PMF curve with the $\mathrm{CG}$ model for $\varepsilon_{L}=8.5 \mathrm{~kJ} / \mathrm{mol}$ (Fig. 5 dashed blue line). The interaction potential is shown in Fig. S7 of the supplementary material. Despite the cost, the electrostatic interactions were calculated with the PME method to capture the repulsion among the charged LK $\alpha 14$ peptides in the long range.

LK $\alpha 14$ aggregates into a tetramer where each peptide adopts an $\alpha$-helix at high peptide concentrations. ${ }^{29}$ To test if our model is stable at larger aggregate sizes, a preformed CG tetramer was simulated using the model with the electrostatic interactions, leucine-leucine nonbonded interactions, and harmonic bonds for restraining the $\alpha$-helix hydrogen bonds. Throughout the simulation, we did not observe disintegration of the tetramer. The RDF analysis of the trajectory is shown in Fig. 4 with the dashed blue line where the match with the AA simulation is fairly good. Interestingly, the height of the peak, which represents the number of neighboring leucine beads, is significantly larger than the AA case. The spherical symmetry of the LJ-cos interaction is partially responsible for the overestimation of the coordination number.

\section{Hydrogen bonds}

Next, we focused on the parameterization of the hydrogen bonding interaction in the CG model. Hydrogen bonds play a crucial role in determining the secondary structure of proteins/peptides and therefore proper representation of this interaction is essential for the success of a CG peptide model. In previous studies, this problem was resolved by explicit representation of the heavy backbone atoms in the CG model and positioning of the hydrogen and oxygen atoms in reference to these. ${ }^{10,31,32,58-61}$ With the donor and acceptor groups defined, a combination of a three body angular potential together with nonbonded pair interactions enables the representation of hydrogen bonds at a CG level.

Since the peptide backbone is represented with only two beads ( $\mathrm{CA}$ and $\mathrm{CN}$ ) in our $\mathrm{CG}$ model and both the acceptor and donor groups are mapped onto the $\mathrm{CN}$ bead, we used an alternative approach. By using the coordinates of the three consecutive beads $\mathrm{CA}_{i+2}, \mathrm{CN}_{i+3}$, and $\mathrm{CA}_{i+3}$, a virtual hydrogen atom $\left(\mathrm{H}_{i+3}\right)$ is positioned [Fig. 6(a)] as described in the mapping section.

Three different criteria are used for the parameterization of the hydrogen bond interaction term: (1) Each acceptor and donor should participate in only one bond at any moment. (2) The interaction should be short ranged and directional; it should lead to an alignment of the participating groups as 
a)
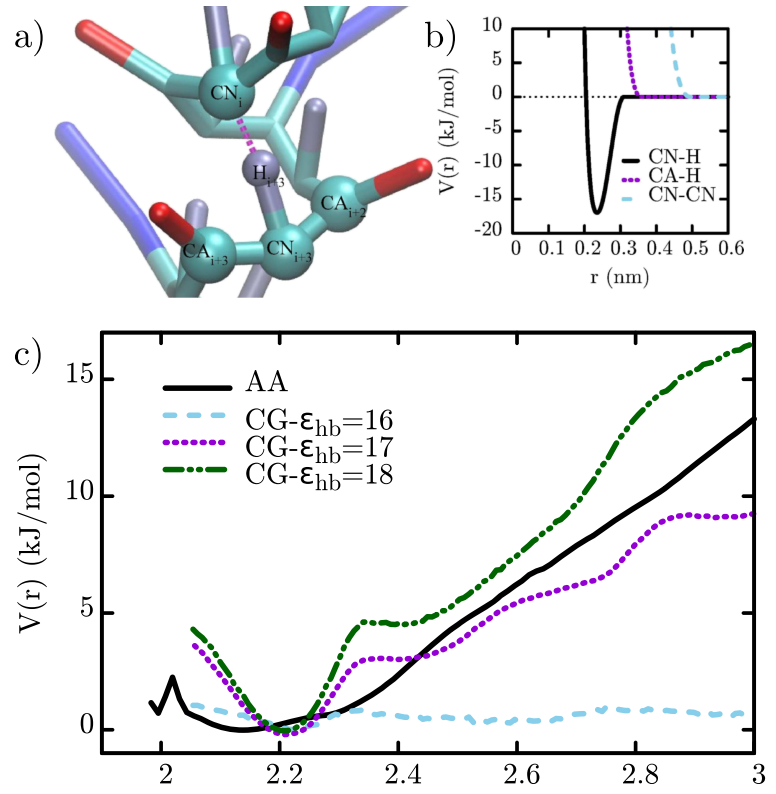

FIG. 6. Hydrogen bonding: (a) Virtual site bead $\mathrm{H}_{i+3}$ is positioned in reference to the beads $\mathrm{CA}_{i+2}, \mathrm{CN}_{i+3}$, and $\mathrm{CA}_{i+3}$. (b) Generic $\mathrm{LJ}$ interactions among $\mathrm{CA}, \mathrm{CN}$, and $\mathrm{H}$ beads determine the hydrogen bond geometry and strength. (c) Comparison of the PMF curves for pulling an $\alpha$-helix from the two ends: AA (solid black line) and CG simulations for three different values of the hydrogen bond strength, $\varepsilon_{h b}$.

depicted in Fig. 6(a). (3) The interaction should be generic, such that any acceptor and donor should be able to form a hydrogen bond if the geometry is suitable. To this aim, three pair interactions are parameterized for representing the hydrogen bonds: two repulsive $(\mathrm{CN}-\mathrm{CN}$ and $\mathrm{CA}-\mathrm{H})$ and one attractive $(\mathrm{CN}-\mathrm{H})$ where the final potentials are shown in Fig. 6(b). The repulsive LJ interactions are parameterized according to Eq. (4) to satisfy the three criteria listed above. The parameters for $\mathrm{LJ}$ repulsion are $\varepsilon_{r}=10 \mathrm{~kJ} / \mathrm{mol}, r_{c u t}$ $=0.495 \mathrm{~nm}$ for $\mathrm{CN}-\mathrm{CN}$, and $r_{c u t}=0.358 \mathrm{~nm}$ for $\mathrm{CA}-\mathrm{H}$. In order to prevent multiple hydrogen bond formation, the nonbonded interactions are excluded for all $\mathrm{CN}_{i}-\mathrm{CN}_{i+2}$, $\mathrm{CN}_{i}-\mathrm{H}_{i+2}$, and $\mathrm{H}_{i}-\mathrm{CN}_{i+2}$ pairs.

For the attractive $\mathrm{CN}-\mathrm{H}$ interaction, the $\mathrm{LJ}-\mathrm{cos}$ form [Eq. (5)] with $r_{m}=0.235 \mathrm{~nm}$ and $r_{\text {cut }}=0.3 \mathrm{~nm}$ is used. Similar to the parameterization of the hydrophobic attraction, hydrogen bonding interaction strength $\left(\varepsilon_{h b}\right)$ can also be parameterized based on a reference AA PMF. In this case, we used the PMF for stretching an $\alpha$-helix from the terminal groups until the molecule is completely extended [Fig. 6(c)]. For the AA PMF curve, the center of mass of NME and ACE caps are used as pull and reference groups, respectively. Since these groups are not represented in the $\mathrm{CG}$ model, $\mathrm{CA}_{0}$ and $\mathrm{CA}_{15}$ are used in the CG PMF calculation. The resulting CG curves are shifted right by $0.16 \mathrm{~nm}$ to account for the difference in the initial distances between the pull and reference groups for AA and CG cases. Since all the hydrogen bonds are broken beyond $3 \mathrm{~nm}$, the PMF curves are shown only up to this distance. The broad minimum in the AA PMF curve is mostly due to the flexibility of the terminal groups. As noted earlier, in bulk water, the $\alpha$-helix conformation is not fully stable for the LK $\alpha 14$ peptide. The molecule displays a variety of structures and as a result, the PMF curve is not very well defined. However, the range from $2.2 \mathrm{~nm}$ up to $2.8 \mathrm{~nm}$ involves consecutive breaking of the hydrogen bonds that stabilize the $\alpha$-helix; hence, we focused only on this portion of the PMF curve.

The CG simulations are performed in the presence of PME electrostatics and leucine-leucine attraction as parameterized in Secs. III D 1 and III D 2. PMF curves for three different values of the interaction strength, $\varepsilon_{h b}$, are shown in Fig. 6 . For $\varepsilon_{h b}=17 \mathrm{~kJ} / \mathrm{mol}$, we observe the closest behavior to the AA case; hence, we chose this value for the hydrogen bonding strength.

\section{Adsorption forces}

Similar to the case where neighboring molecules stabilize the $\alpha$-helix, a hydrophobic/hydrophilic interface, such as air/water, also stabilizes the $\alpha$-helix conformation for LK $\alpha 14 .{ }^{35,36}$ In our implicit water CG model to mimic a slab of water, identical to the AA simulation of the air/water interface, two walls are added at the top and bottom of the simulation box. Due to the presence of the walls, the periodic boundary conditions are applied only in the x-y plane.

The air/water interface leads to the partitioning of the LK $\alpha 14$ peptide's side chains, such that the leucines are pushed towards the air and lysines remain on the water side of the interface. ${ }^{36}$ Hence, we would like to have a soft interface that is permeable to hydrophobic beads and impermeable to hydrophilic ones. In order to mimic this partitioning effect, we relied on a set of interaction potentials (shown in Fig. 7) between the CG beads and the walls. Here, we will use a coordinate system where the interface and the top wall are positioned at $z=0$ and $z=1 \mathrm{~nm}$, respectively. The charged $\mathrm{KN}$ beads are repelled from the interface with a repulsive $\mathrm{LJ}$ potential [Eq. (4) with $\varepsilon_{r}=1.0 \mathrm{~kJ} / \mathrm{mol}$ and $r_{c u t}=1.4 \mathrm{~nm}$ measured from the wall] shown with the dashed blue line in Fig. 7. The $\mathrm{L}$ beads are attracted towards the interface starting at $z_{a}=-0.6 \mathrm{~nm}$ below the interface and the attractive force is zero above the interface (on the air side),

$$
U= \begin{cases}0, & z<z_{a}, \\ -\varepsilon_{w}\left[\cos \left(\frac{\pi z}{2 z_{a}}\right)\right]^{2}, & z_{a}<z<0, \\ -\varepsilon_{w}, & z>0,\end{cases}
$$

where $\varepsilon_{w}$ is the strength of the attractive potential, which will be discussed below. The range of the attraction, $z_{a}$, is chosen as half the width of the interface region in the AA simulation (see Fig. S8 of the supplementary material). The remaining beads do not interact with the interface. In order to prevent any particles from going outside the simulation box, a repulsive LJ interaction [Eq. (4) with $\varepsilon_{r}=10 \mathrm{~kJ} / \mathrm{mol} r_{\text {cut }}=0.495 \mathrm{~nm}$, where the distance is measured from the wall position] is used as shown in Fig. 7(a) with the dotted purple line. The interface at the bottom of the water slab is represented in an identical manner. The wall interactions are parameterized to reproduce the AA density profiles for the CG backbone and side chain beads, where the comparison is shown in Fig. 7(b).

The final parameter to be determined is the strength of the attraction on the $\mathrm{L}$ beads towards the interface, $\varepsilon_{w}$, for which we used the AA PMF curve for adsorption of the LK $\alpha 14$ 

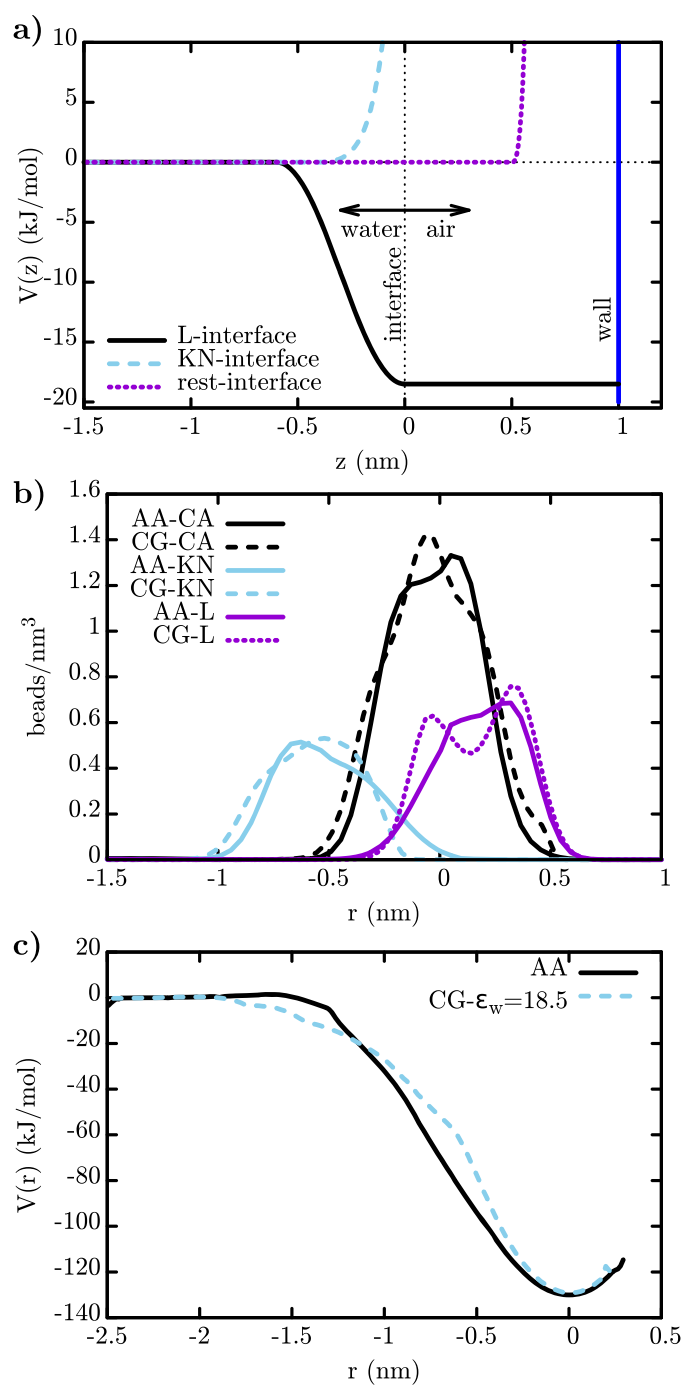

FIG. 7. (a) Interface interaction potentials for L (solid black line), KN (dashed blue line), and the remaining CG beads (dotted purple line). The $z$ distance is measured from the interface and the top wall is positioned at $z=1 . \mathrm{nm}$. (b) Density profiles for backbone (CA), leucine (L), and lysine (KN) side chains at the CG interface in comparison to AA results. (c) Comparison of AA and CG PMFs for pulling the $\mathrm{LK} \alpha 14$ peptide from the interface towards water. The CG model matches perfectly with the adsorption strength of leucine beads set to $\varepsilon_{w}=18.5 \mathrm{~kJ} / \mathrm{mol}$.

peptide to the air/water interface. ${ }^{36}$ In Fig. 7(c), the reference AA PMF curve and the matching CG analog, obtained with $\varepsilon_{w}=18.5 \mathrm{~kJ} / \mathrm{mol}$, are shown. The value of $\varepsilon_{w}=18.5$ is obtained by iterating until a good match to the AA PMF curve is obtained. During the trials, the hydrogen bonds were restrained to maintain the helical conformation. These restraints are removed after obtaining the $\varepsilon_{w}$ value and the dashed blue line in Fig. 7 represents the CG result with PME electrostatics, leucine attraction, and hydrogen bond strength as parameterized in Secs. III D 1-III D 3.

\section{PERFORMANCE OF THE CG MODEL}

In this section, by using the final set of parameters for the CG model, we will analyze its performance in terms of structural, dynamic, and thermodynamic properties in comparison to the AA results and experimental evidence.

\section{A. A single LKa14 peptide in bulk water}

In order to analyze the influence of the hydrogen bond strength on the structural dynamics and conformational heterogeneity of the peptide, we performed a Hamiltonian replica exchange (HREX) simulation. For the HREX simulation, 16 replicas were used where the replicas differ for the strength of the hydrogen bonding interaction, ranging from $\varepsilon_{h b}=14$ up to $\varepsilon_{h b}=17.75 \mathrm{~kJ} / \mathrm{mol}$ with increments of $0.25 \mathrm{~kJ} / \mathrm{mol}$. Convergence of the HREX simulation was validated by block averaging and the mixing efficiency was checked by generating an exchange matrix which can be seen in Fig. S9 of the supplementary material. Resulting trajectories are analyzed for the probability distribution of the $\psi$ backbone dihedral angle in Fig. 8. The AA reference distributions from a single peptide in vacuum, in bulk water, and within a tetramer are also shown in the figure. For a quantitative analysis of the results, the left-hand side probability $[\operatorname{Prob}(\psi<0)]$ is provided next to the line labels for both the AA references and the $\mathrm{CG}$ replicas. From the CG simulation, only the results of the replicas from $\varepsilon_{h b}=16.25$ up to $\varepsilon_{h b}=17.25 \mathrm{~kJ} / \mathrm{mol}$ are shown. Among these the closest match to the AA bulk simulation
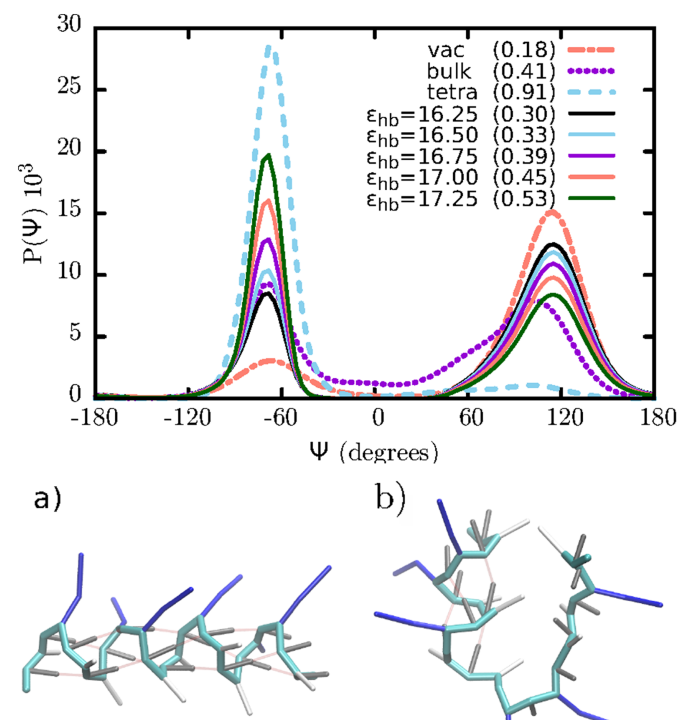

AA: $22 \%$ CG: $20 \%$

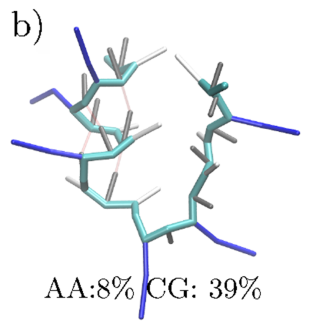

c)
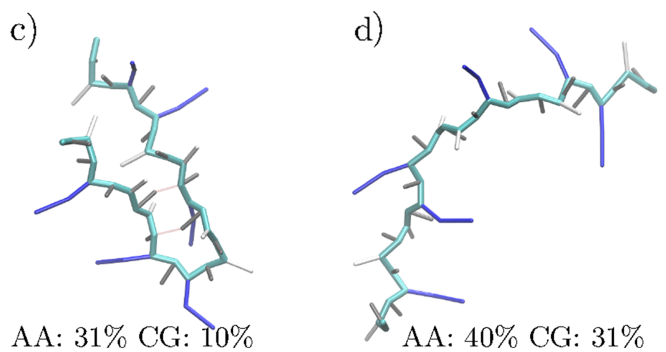

FIG. 8. Probability distribution for the backbone dihedral $\psi$ for the AA reference simulations (vacuum, bulk water, and within a tetramer) and CG HREX simulations for replicas with $\varepsilon_{h b}$ values ranging from 16.25 to $17.25 \mathrm{~kJ} / \mathrm{mol}$. The probabilities for $\psi<0$ are shown next to the labels for each case. The distinct conformations adopted by the CG peptide for $\varepsilon_{h b}=17.0 \mathrm{~kJ} / \mathrm{mol}$, shown at the bottom, display a remarkable similarity to the AA case. ${ }^{36}$ For each conformation percentages of frames in AA REX and CG HREX simulations, as obtained from the cluster analysis, are shown as well. 
$[\operatorname{Prob}(\psi<0)=0.41]$ is obtained by $\varepsilon_{h b}=16.75[\operatorname{Prob}(\psi$ $<0)=0.39]$ and $17.0 \mathrm{~kJ} / \mathrm{mol}[\operatorname{Prob}(\psi<0)=0.45]$. Hence, our choice $\varepsilon_{h b}=17 \mathrm{~kJ} / \mathrm{mol}$ also produces a good representation for the backbone dihedral probability distribution.

As noted earlier, the AA simulations reveal that the LK $\alpha 14$ peptide displays a rich conformational dynamics in bulk water, where complete $\alpha$-helix, $\beta$-hairpin, half- $\alpha$-helix, and random conformations were listed as the dominant secondary structures. ${ }^{36}$ In Fig. 8, conformations obtained from the HREX replica with $\varepsilon_{h b}=17 \mathrm{~kJ} / \mathrm{mol}$ are shown. Remarkably, all the aforementioned secondary structures for the AA simulation are also observed in the CG case. In order to compare the weight of these distinct conformations in the phase space of AA and CG simulations, we also performed a cluster analysis. The percentages for each four categories are given in Fig. 8. The CG model captures the $\alpha$-helix propensity correctly. The AA simulations display a variety of $\beta$-hairpin conformations (with arbitrary turn positions along the sequence). The CG model underestimates the $\beta$-hairpin conformation. On the other hand, the CG model strongly overestimates the half- $\alpha$-helix conformation.

In order to study the kinetics and folding/unfolding dynamics of the CG model, we also performed simulations of an isolated single CG LK $\alpha 14$ peptide with standard MD. Two different simulations with initial conditions chosen as an $\alpha$ helix and an extended conformation were performed. In order to trace the secondary structure of each individual residue, we analyzed the hydrogen bond formation/breaking events during the simulation. Similar to the secondary structure analysis by DSSP, ${ }^{62}$ this enables us to observe the conformation of all the residues simultaneously. As shown in Fig. 2, when the dihedral angle adopts the $\alpha$-helix conformation, the $\mathrm{CN}_{i+3}-\mathrm{CN}_{i}$ distances drop below $0.55 \mathrm{~nm}$. In Fig. 9, for each of the 12 possible $\alpha$-helix characteristic hydrogen bonds, a color block is used if the bond is intact. Hence at any given time if all the bonds are shown in color, this suggests a full $\alpha$-helix and for a half- $\alpha$-helix, only a group of the bonds are shown in color. This analysis only traces the $\alpha$-helix secondary structure formation, leaving all other possible structures in a single group. The timeline data in Fig. 9 clearly show that the CG peptide displays multiple folding/unfolding events independent of the initial starting conformation. The time scale of the conformational transitions is similar to the AA case [see Fig. 1(c) in the work of Dalgicdir et al. ${ }^{35}$ and Fig. 2 in the work of Dalgicdir and Sayar $^{36}$ ], which is surprising considering that CG models usually have accelerated dynamics due to softer interaction potentials compared to the AA case. As shown in a recent study, ${ }^{63}$ similar to the structural and thermodynamic properties, recapturing the kinetics of the underlying AA system is also highly dependent on the specific parameterization of the CG model.

\section{B. Aggregation in bulk water}

For the parameterization of the leucine-leucine hydrophobic attraction term, the hydrogen bonds were restrained in order to preserve the $\alpha$-helix conformation (Sec. III D 2). We repeated the umbrella sampling simulation for splitting the dimer using the final CG model in order to validate the
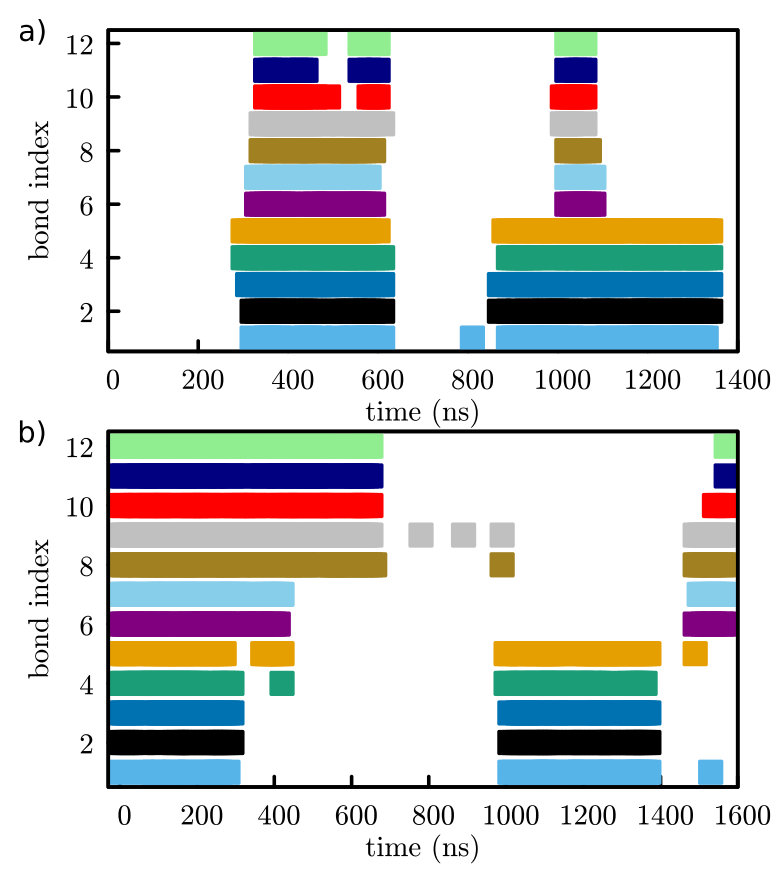

FIG. 9. Time evolution of the characteristic $\alpha$-helix hydrogen bonds: For each of the 12 possible bonds if the bond is intact (i.e., $\mathrm{CN}_{i+3}-\mathrm{CN}_{i} \leq 0.55 \mathrm{~nm}$ ), a color block is used. Independent of the initial conformation [extended (a) and $\alpha$-helix (b)], the peptide displays a dynamic conformational behavior with multiple folding/unfolding transitions. A movie of the trajectories for both initial conditions is available as the supplementary material.

use of restraints. The resulting PMF curve is shown in Fig. 5 with a dotted purple line. The PMF curve is almost identical to the CG case with the restraints, except for the region around the PMF minimum. In this region, as the restraining force for the umbrella simulation forces the molecules to closer distances, one of the peptides responds by unfolding (see Fig. 5 inset). This partial unfolding both eliminates strong forces due to the umbrella simulation restraint and also helps the dimer to reduce its hydrophobic contact area. Nevertheless, we can conclude that the CG model with the hydrogen bond parameterization still yields a similar PMF profile for the dimer.

AA simulations suggest a drastic increase in the stability of the $\alpha$-helix conformation upon dimerization. ${ }^{36}$ In an analogous manner, we set up a simulation where two peptides are placed in the box with no initial contact. One of the peptides was restrained to the $\alpha$-helix conformation, by doubling the hydrogen bonding interaction strength, to act as a molecular template for the second molecule. The second molecule was kept free (i.e., without any restraints) and was initially put in an extended conformation [Fig. 10(b) left].

The time evolution of the secondary structure of the free $\mathrm{LK} \alpha 14$ can be analyzed via the formation of characteristic $\alpha$-helix hydrogen bonds as shown in Fig. 10(a). The initial extended conformation evolves into a partial $\alpha$-helix around $90 \mathrm{~ns}$. Around $100 \mathrm{~ns}$, the molecules merge and remain in contact for the remainder of the simulation [center-tocenter distance for the peptides is shown in Fig. 10(a) with red dots and second y-axis on the right]. Complete folding of the free peptide requires an additional $250 \mathrm{~ns}$, upon which the free peptide adopts the $\alpha$-helix conformation and 


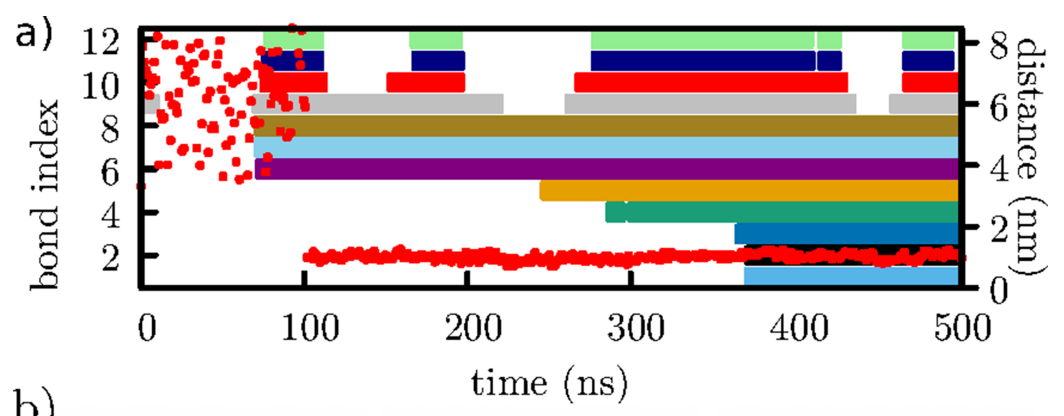

b)

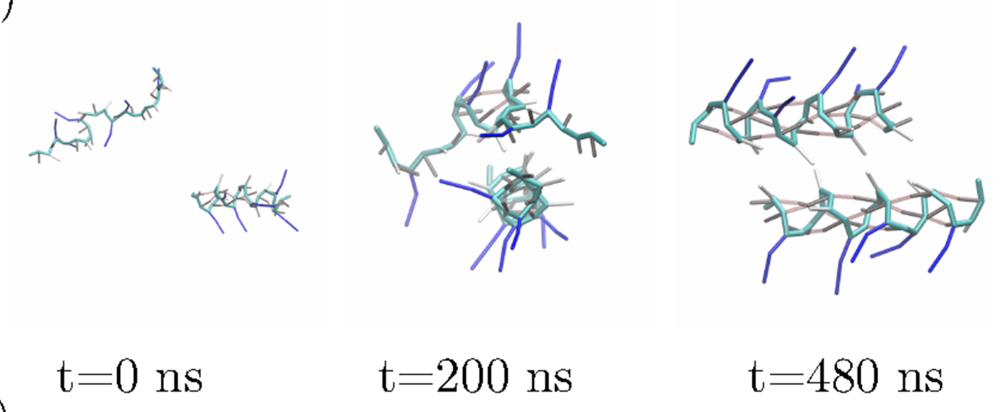

c)
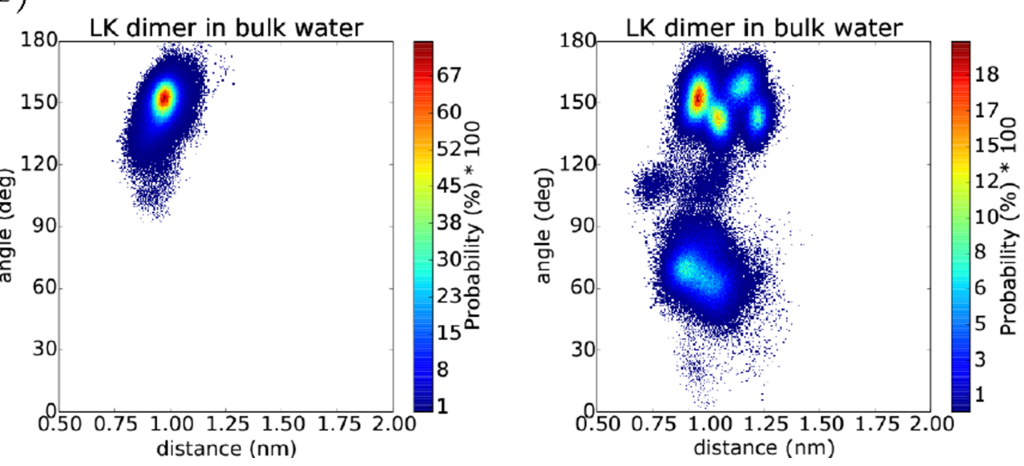

FIG. 10. Folding of an LK $\alpha 14$ peptide in the presence of a neighboring $\mathrm{LK} \alpha 14$ (with hydrogen bond restraints to maintain the $\alpha$-helix conformation). (a) Hydrogen bonds formed and the center-to-center distance between the peptides (second y-axis) as a function of time. (b) Snapshots from the simulation. (c) Comparison of the distance vs. angle correlation plot for the AA (left) and CG (right) simulations of the dimer. A movie of the dimerization and folding trajectory in (a) is available as the supplementary material.

maintains this conformation except for partial unfolding of the termini.

Even though the two peptides remain as a dimer and maintain their $\alpha$-helix conformation, the orientation of the peptides with respect to each other still displays a large amount of flexibility in the CG simulation. In order to quantify this flexibility and compare it with the AA simulation, we extended the simulation of the dimer for an additional $500 \mathrm{~ns}$. In this case, both of the peptides were left free, i.e., without any restraints to enforce the $\alpha$-helix conformation. The correlation plot for the distance vs. angle between the two peptides is shown in Fig. 10(c). The AA and CG correlation plots are shown on the left and right, respectively. Since the terminal residues are rather flexible and could distort the center-to-center distance calculations, analyses were performed on the eight residues at the center of the $\mathrm{LK} \alpha 14$ sequence. Similarly, the angle between the helical axes of the two peptides is calculated by fitting a vector to the backbone beads of the eight central residues for each of the peptides. The AA simulations reveal that both the parallel and anti-parallel orientations of the peptides are stable and transitions from one to the other are extremely rare. ${ }^{36}$ The AA correlation plot shown in Fig. 10(c) on the left belongs to the anti-parallel orientation which remains stable for the $1 \mu \mathrm{s}$ simulation. The CG model displays a more flexible dynamics, where the two peptides explore a broad range of angular orientations. Nevertheless the anti-parallel orientation remains the most populated state, with distance and angle exactly matching the AA simulation. Further analysis of the remaining equilibrium points in the correlation plot of $\mathrm{CG}$ simulation is provided in Fig. S12 of the supplementary material.

\section{Adsorption and aggregation at the interface}

The interaction parameters for the CG peptide's behavior at the interface were based on the density profile of the corresponding beads and the PMF curve for adsorption of the peptide to the interface in Sec. III D 4. In order to test the conformational behavior of the CG model during adsorption, we set up a simulation where an LK $\alpha 14$ peptide, initially in the extended conformation, is simulated in a box with walls at the top and bottom to mimic a water slab identical to the AA simulations. ${ }^{36}$ The peptide makes the initial contact with the bottom interface in a random conformation [Fig. 11(b) left]. The time evolution of the characteristic $\alpha$-helix hydrogen bonds is shown in Fig. 11(a). Within 100 ns, half of the peptide folds, and the folding to the full $\alpha$-helix is completed around $200 \mathrm{~ns}$. For the remainder of the simulation, the molecule remains at the interface in this stable secondary structure. Hence, our CG model not only reproduces the correct density and PMF profile but also represents the folding 


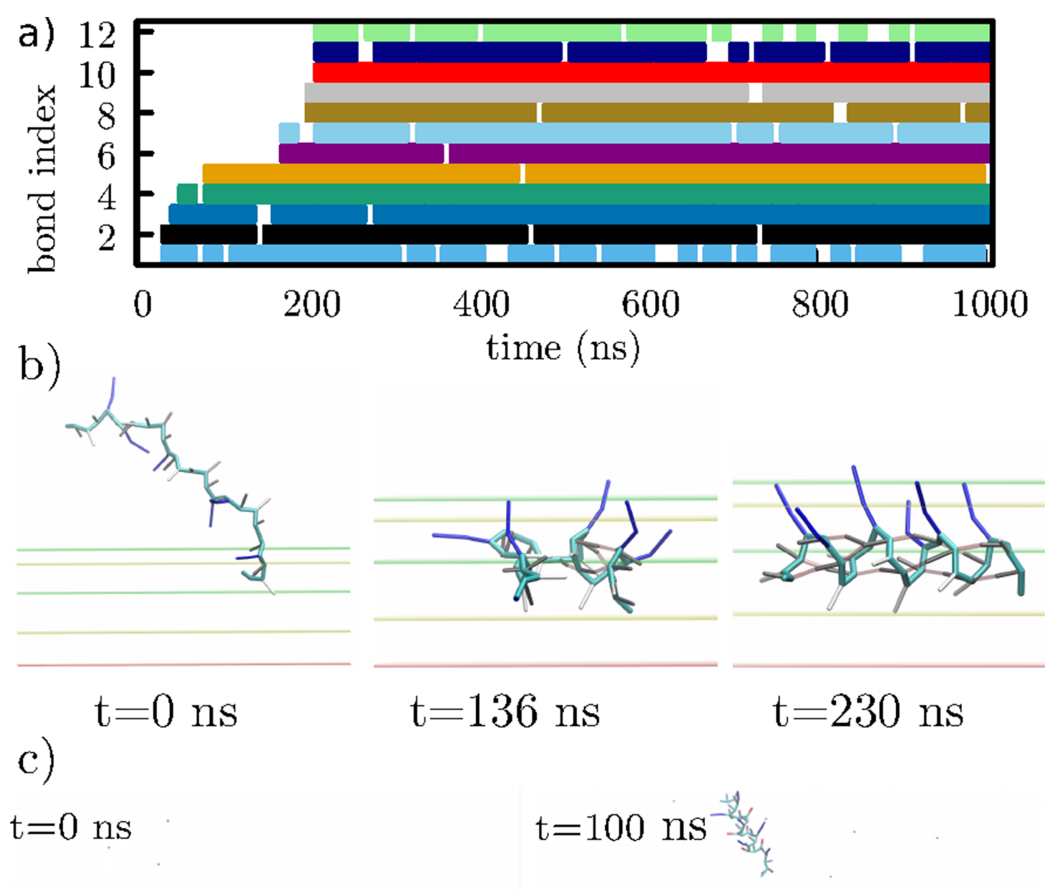

FIG. 11. Adsorption of a CG peptide to the interface: (a) Timeline analysis of the hydrogen bond formation. (b) Peptide adsorbs to the interface in an unfolded conformation and adopts the $\alpha$-helix conformation at the interface. (c) Eight molecules initially aligned as a stripe, split up to form dimers and trimers analogous to the AA simulations. $^{36}$
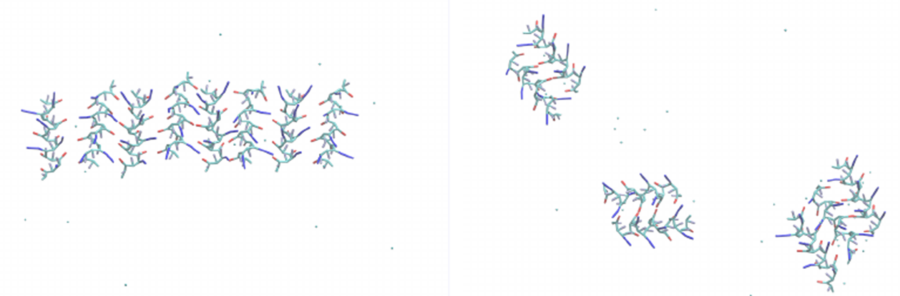

and enhanced stability of the $\alpha$-helix conformation at the interface.

Another observation in our AA simulations ${ }^{36}$ was that unlike the bulk system, where tetramer is the equilibrium aggregate size, at the interface, the enforced orientation of the lysine residues increases the repulsion between the LK $\alpha 14$ peptides. As a result, aggregation at the interface is limited to two to three molecules. We used a system with eight LK $\alpha 14$ peptides, aligned in the antiparallel orientation in the form of a stripe at the interface, to test the aggregation characteristics of the CG model. The initial stripe of eight LK $\alpha 14 \mathrm{~s}$ [Fig. 11(c) left] immediately falls apart and the molecules display a dynamic assembly disassembly behavior at the interface. All of the molecules remained at the interface throughout the simulation. Dimers and trimers were observed; however, aggregates of larger size were prohibited with the increased repulsion between the charged lysine side chains, in a similar manner to the AA simulations.

\section{CONCLUSIONS}

In the development of a CG model for peptides, two important decisions define much of the final properties of the model. The mapping from the atomistic to the CG model is the first major step. In many cases, this mapping is shaped by the aim of the CG model and the intuition of the researcher. Once the resolution of the $\mathrm{CG}$ model is set, the next important decision is concerned with the distribution of the forces to the bonded and nonbonded interaction terms involved.

In our model, bonded interactions were intentionally designed to be unbiased with respect to the conformational behavior of the molecule. We have used this approach earlier in our study of the self-assembly of a triblock peptide into one-dimensional aggregates ${ }^{27}$ and for the representation of $\mathrm{pH}$ dependent conformational helix-coil transition of a peptide oligomer (EALA). ${ }^{26}$ In both of these studies, except for the specific side chain bonded interaction terms, the basis for the backbone interactions were identical. The fundamental difference among the triblock, EALA and LK $\alpha 14$ CG models is the parameterization of the nonbonded interactions. For the triblock peptide $\mathrm{CG}$ model, we have relied on target radial distribution functions. In the case of the EALA molecule, the parameterization was done in a phenomenological manner to capture the helix-coil transition.

In the current study, we demonstrated that when the nonbonded interactions are parameterized based on carefully designed potential of mean force calculations, one can generate a multi-state $\mathrm{CG}$ model which is capable of representing both the conformational heterogeneity and the aggregation characteristics of the underlying atomic system. The virtual bond representation for hydrogen bond formation is in particular promising. Despite the fact that none of the AA reference 
states had a particular hint of $\beta$-sheet formation and hydrogen bonds were optimized to represent the $\alpha$-helix structure, our CG model was capable of capturing the $\beta$-hairpin formation at a single molecule level. However, the $\beta$-sheet formation propensity of our CG model is still weak compared to the AA case.

The success of our CG model is largely based on the ideal characteristics of the LK $\alpha 14$ peptide. The two types of residues contribute to distinct molecular forces in this molecule. The lysine side chains predominantly disfavor $\alpha$ helix formation via Coulomb repulsion, whereas the leucine side chains drive the molecule towards compact conformations. Peptides of biological origin are not so simple. Even for many synthetic peptides, such a straightforward mapping of the molecular forces from the AA to the CG model is not possible. For example, the glutamic acid side chains in the EALA molecule not only possess a negative charge but also play an important role in destabilizing the $\alpha$-helix conformation with their tendency to form side chain-backbone hydrogen bonds.

The CG model enables approximately a 100 fold increase in speed, thanks to its implicit solvent parameterization. This acceleration is crucial in order to explore aggregation characteristics of the molecule in general. An important advantage of our multi-state CG model compared to earlier studies on assembly of helical peptides ${ }^{32}$ is the realistic representation of the conformational heterogeneity of the molecule. This property will be particularly important if one wants to use such CG models to study intrinsically disordered peptides.

Many open questions remain in regard to the transferability issue. In the current study, we have focused on the transferability of the CG model to capture the correct conformational dynamics and aggregation behavior of a specific peptide. It remains to be seen whether this approach can be generalized to other peptide molecules, i.e., to achieve sequence-wise transferability. In theory, one can mutate the LK sequence and generate the required AA PMF curves to obtain the parameters for other amino acids, which we are currently working on. In addition, conformational transitions in response to molecular and macroscopic interfaces are just one of the possible triggering mechanisms. We also hope to study temperature, salt, and concentration dependence of our CG model in the future.

\section{SUPPLEMENTARY MATERIAL}

See supplementary material for the probability distributions for bonded interactions; bonded and nonbonded interaction potentials for the final CG model; Boltzmann inversion for RDF of leucine side chains; water density profile in AA simulation for the air/water interface; mixing efficiency matrix for HREX simulation; movie for conformational dynamics of a single $\mathrm{LK} \alpha 14$ in bulk water; movie for folding of an $\mathrm{LK} \alpha 14$ in the presence of a neighboring molecule; and finally for the analysis of the dimer structure in bulk water. In addition, the full set of files required for the simulation of a CG peptide in bulk water, at the air/water interface, and in a HREX simulation is provided as a tar file.

\section{ACKNOWLEDGMENTS}

The authors thank TUBITAK for funding (Grant No. 212T184). M. Sayar thanks the Turkish Academy of Sciences for financial support through the Young Scientist Award Program (No. TUBA GEBIP 2012 awardee). We are also thankful to Dr. Beytullah Özgür for a critical reading of our manuscript and fruitful scientific discussions.

${ }^{1}$ J. J. Sakon and K. R. Weninger, Nat. Methods 7, 203 (2010).

${ }^{2}$ M. F. Juette, D. S. Terry, M. R. Wasserman, Z. Zhou, R. B. Altman, Q. Zheng, and S. C. Blanchard, Curr. Opin. Chem. Biol. 20, 103 (2014).

${ }^{3}$ I. König, A. Zarrine-Afsar, M. Aznauryan, A. Soranno, B. Wunderlich, F. Dingfelder, J. C. Stüber, A. Plückthun, D. Nettels, and B. Schuler, Nat. Methods 12, 773 (2015).

${ }^{4}$ J.-N. Longchamp, S. Rauschenbach, S. Abb, C. Escher, T. Latychevskaia, K. Kern, and H.-W. Fink, Proc. Natl. Acad. Sci. U. S. A. 114, 1474 (2017).

${ }^{5}$ T. Schlick, R. Collepardo-Guevara, L. A. Halvorsen, S. Jung, and X. Xiao, Q. Rev. Biophys. 44, 191 (2011).

${ }^{6}$ W. Noid, J. Chem. Phys. 139, 090901 (2013).

${ }^{7}$ M. Khalili, A. Liwo, F. Rakowski, P. Grochowski, and H. A. Scheraga, J. Phys. Chem. B 109, 13785 (2005).

${ }^{8}$ S. J. Marrink, A. H. de Vries, and A. E. Mark, J. Phys. Chem. B 108, 750 (2004).

${ }^{9}$ J. Maupetit, P. Tuffery, and P. Derreumaux, Proteins 69, 394 (2007).

${ }^{10}$ T. Bereau and M. Deserno, J. Chem. Phys. 130, 235106 (2009).

${ }^{11}$ S. M. Gopal, S. Mukherjee, Y.-M. Cheng, and M. Feig, Proteins 78, 1266 (2010).

${ }^{12}$ M. Cheon, I. Chang, and C. K. Hall, Proteins 78, 2950 (2010).

${ }^{13}$ P. Kar and M. Feig, Adv. Protein Chem. Struct. Biol. 96, 143 (2014).

${ }^{14}$ G. O. Rutter, A. H. Brown, D. Quigley, T. R. Walsh, and M. P. Allen, Phys. Chem. Chem. Phys. 17, 31741 (2015).

${ }^{15}$ M. Seo, S. Rauscher, R. Pomès, and D. P. Tieleman, J. Chem. Theory Comput. 8, 1774 (2012).

${ }^{16}$ F. Sterpone, S. Melchionna, P. Tuffery, S. Pasquali, N. Mousseau, T. Cragnolini, Y. Chebaro, J.-F. St-Pierre, M. Kalimeri, A. Barducci, Y. Laurin, A. Tek, M. Baaden, P. H. Nguyen, and P. Derreumaux, Chem. Soc. Rev. 43, 4871 (2014)

${ }^{17}$ O. Engin, A. Villa, C. Peter, and M. Sayar, Macromol. Theory Simul. 20, 451 (2011).

${ }^{18}$ E. C. Allen and G. C. Rutledge, J. Chem. Phys. 128, 154115 (2008).

${ }^{19}$ S. Izvekov, P. W. Chung, and B. M. Rice, J. Chem. Phys. 133, 064109 (2010).

${ }^{20}$ A. Lyubartsev, A. Mirzoev, L. Chen, and A. Laaksonen, Faraday Discuss. 144, 43 (2009).

${ }^{21}$ J.-W. Shen, C. Li, N. F. van der Vegt, and C. Peter, J. Chem. Theory Comput. 7, 1916 (2011).

${ }^{22}$ E. Brini and N. F. A. van der Vegt, J. Chem. Phys. 137, 154113 (2012).

${ }^{23}$ C. Dalgicdir, O. Sensoy, C. Peter, and M. Sayar, J. Chem. Phys. 139, 234115 (2013).

${ }^{24}$ M. Jochum, D. Andrienko, K. Kremer, and C. Peter, J. Chem. Phys. 137, 064102 (2012).

${ }^{25}$ T. C. Moore, C. R. Iacovella, and C. McCabe, J. Chem. Phys. 140, 224104 (2014).

${ }^{26}$ C. Dalgicdir, C. Globisch, M. Sayar, and C. Peter, Eur. Phys. J. Spec. Top. 225, 1463 (2016).

${ }^{27}$ B. Ozgur and M. Sayar, J. Phys. Chem. B 120, 10243 (2016).

${ }^{28}$ F. Ding, J. M. Borreguero, S. V. Buldyrey, H. E. Stanley, and N. V. Dokholyan, Proteins 53, 220 (2003).

${ }^{29}$ W. F. DeGrado and J. D. Lear, J. Am. Chem. Soc. 107, 7684 (1985).

${ }^{30}$ O. Mermut, D. C. Phillips, R. L. York, K. R. McCrea, R. S. Ward, and G. A. Somorjai, J. Am. Chem. Soc. 128, 3598 (2006).

${ }^{31}$ A. V. Smith and C. K. Hall, J. Mol. Biol. 312, 187 (2001).

${ }^{32}$ A. V. Smith and C. K. Hall, Proteins 44, 376 (2001).

${ }^{33}$ J. E. Baio, A. Zane, V. Jaeger, A. M. Roehrich, H. Lutz, J. Pfaendtner, G. P. Drobny, and T. Weidner, J. Am. Chem. Soc. 136, 15134 (2014).

${ }^{34}$ H. Lutz, V. Jaeger, M. Bonn, J. Pfaendtner, and T. Weidner, J. Pept. Sci. 23, 141 (2017).

${ }^{35}$ C. Dalgicdir, C. Globisch, C. Peter, and M. Sayar, PLoS Comput. Biol. 11, e1004328 (2015).

${ }^{36}$ C. Dalgicdir and M. Sayar, J. Phys. Chem. B 119, 15164 (2015). 
${ }^{37}$ S. Pronk, S. Páll, R. Schulz, P. Larsson, P. Bjelkmar, R. Apostolov, M. R. Shirts, J. C. Smith, P. M. Kasson, D. van der Spoel, B. Hess, and E. Lindahl, Bioinformatics 29, 845 (2013).

${ }^{38}$ G. Bussi, D. Donadio, and M. Parrinello, J. Chem. Phys. 126, 014101 (2007).

${ }^{39}$ U. Essmann, L. Perera, M. L. Berkowitz, T. Darden, H. Lee, and L. G. Pedersen, J. Chem. Phys. 103, 8577 (1995).

${ }^{40}$ N. Schmid, A. P. Eichenberger, A. Choutko, S. Riniker, M. Winger, A. E. Mark, and W. F. van Gunsteren, Eur. Biophys. J. 40, 843 (2011).

${ }^{41}$ H. J. C. Berendsen, J. R. Grigera, and T. P. Straatsma, J. Phys. Chem. 91, 6269 (1987).

${ }^{42}$ V. Rühle and C. Junghans, Macromol. Theory Simul. 20, 472 (2011).

${ }^{43}$ G. M. Torrie and J. P. Valleau, Chem. Phys. Lett. 28, 578 (1974).

${ }^{44}$ J. G. Kirkwood, J. Chem. Phys. 3, 300 (1935).

${ }^{45}$ S. Kumar, J. M. Rosenberg, D. Bouzida, R. H. Swendsen, and P. A. Kollman, J. Comput. Chem. 16, 1339 (1995).

${ }^{46}$ J. S. Hub, B. L. De Groot, and D. Van Der Spoel, J. Chem. Theory Comput. 6, 3713 (2010).

${ }^{47}$ G. Bussi, Mol. Phys. 112, 379 (2014).

${ }^{48}$ B. Hess, C. Kutzner, D. Van Der Spoel, and E. Lindahl, J. Chem. Theory Comput. 4, 435 (2008).

${ }^{49}$ M. Bonomi, D. Branduardi, G. Bussi, C. Camilloni, D. Provasi, P. Raiteri, D. Donadio, F. Marinelli, F. Pietrucci, R. A. Broglia et al., Comput. Phys. Commun. 180, 1961 (2009).
${ }^{50}$ C. D. Schwieters and G. M. Clore, J. Magn. Reson. 149, 239 (2001).

${ }^{51}$ G. Bellesia and J.-E. Shea, J. Chem. Phys. 126, 245104 (2007).

${ }^{52}$ E. Lindahl, A. Hess, R. van Buuren, E. Apol, P. Meulenhoff, D. Tieleman, A. Sijbers, K. Feenstra, R. van Drunen, and H. Berendsen, Gromacs User Manual, Version 4.5. 6., 2010.

${ }^{53}$ W. Tschöp, K. Kremer, J. Batoulis, T. Bürger, and O. Hahn, Acta Polym. 49, 61 (1998).

${ }^{54}$ V. Harmandaris, N. Adhikari, N. F. van der Vegt, and K. Kremer, Macromolecules 39, 6708 (2006).

${ }^{55}$ J. C. Jo and B. C. Kim, Bull. Korean Chem. Soc. 21, 419 (2000).

${ }^{56}$ T. Soddemann, B. Dünweg, and K. Kremer, Eur. Phys. J. E 6, 409 (2001).

${ }^{57}$ D. Reith, M. Pütz, and F. Müller-Plathe, J. Comput. Chem. 24, 1624 (2003).

${ }^{58}$ A. Voegler Smith and C. K. Hall, Proteins 44, 344 (2001).

${ }^{59}$ D. Klimov, M. Betancourt, and D. Thirumalai, Folding Des. 3, 481 (1998).

${ }^{60}$ E.-H. Yap, N. L. Fawzi, and T. Head-Gordon, Proteins: Struct., Funct., Bioinf. 70, 626 (2008).

${ }^{61}$ A. Irbäck, F. Sjunnesson, and S. Wallin, Proc. Natl. Acad. Sci. U. S. A. 97, 13614 (2000).

${ }^{62}$ W. Kabsch and C. Sander, Biopolymers 22, 2577 (1983).

${ }^{63}$ J. F. Rudzinski, K. Kremer, and T. Bereau, J. Chem. Phys. 144, 051102 (2016). 